\title{
Concepção de saúde de usuários da Estratégia Saúde da Família e novo modelo assistencial
}

Pesquisa de abordagem qualitativa que buscou identificar as concepções de saúde de usuários da Estratégia Saúde da Família (ESF), analisando aproximações com a biomedicina ou com um novo modelo assistencial em saúde. Os dados coletados por meio de entrevistas semiestruturadas mostraram que saúde não é apenas ausência de doença; são necessárias qualidade de vida e bem viver, autonomia para ações cotidianas e boas condições econômicas. A visão dos usuários aproximou-se das diretrizes da ESF, da Política Nacional de Promoção da Saúde e da Safety Promotion/ OMS. Conclui-se que a expectativa dos usuários deve ser considerada no planejamento em saúde.

Descritores: Assistência Integral à Saúde, Sistemas de Saúde, Assistência ao Paciente, Saúde da Família, Atenção Básica.

\section{Conception of health of the of Family Health Strategy users and new health care model}

This is a research of qualitative approach, with the purpose to identify the conceptions of health of the Family Health Strategy users analyzing approaches with the biomedicine or with a new health care model. The data collected through semi-structured interviews showed that health is not only the absent of disease, it is necessary to have quality of life and well being, autonomy for daily activities and good economic conditions the users' view close to the Brazilian Family Health Strategy, to the Politics of Health Promotion and to the WHO' Safety Promotion. We conclude that users' comprehension of health should be considered in the health care organizations.

Descriptors: Comprehensive Health Care model, Health System, Patient Care, Family Health, Primary Health Care.

\section{Concepción de salud de usuarios de la Estrategia Salud de la Familia y nuevo modelo asistencial}

Se trata de investigación cualitativa que busca identificar las concepciones de salud de usuarios de la Estrategia Salud de la Familia (ESF) analizando aproximaciones con los presupuestos de la biomedicina o con un nuevo modelo asistencial en salud. Los datos obtenidos por entrevistas semiestructuradas mostrarán que salud no es apenas la ausencia de enfermedad; es necesario calidad de vida y bien vivir, autonomía para las actividades cotidianas y buenas condiciones económicas. La visión de los usuarios está próxima de la ESF, de la Política Nacional de Promoción de Salud y de la Safety Promotion (OMS). Se concluyó que se considere la expectativa de los usuarios en el planeamiento en salud.

Descriptores: Atención Integral de Salud, Sistemas de Salud, Atención al Paciente, Salud de la Familia, Atención Primaria de Salud.

\section{INTRODUÇÃO}

A $S$ diferentes concepções de saúde-doença são históricas e culturais e influenciam o modelo e as práticas assistenciais em saúde e enfermagem. As ideias sobre saúde e doença variaram de acordo com o entendimento que as populações e comunidades tiveram do mundo, da doença e do processo de viver, e têm associação com valores ético-morais ${ }^{(1-3)}$. Não existe representação universal de saúde e doença, há diferentes percepções influenciadas pela cultura e situação de classe. A importância e os significados dados ao adoecer são um processo particular, relacionado às experiências individuais ${ }^{(2)}$.

A concepção de saúde que orienta a biomedicina teve origem na ciência moderna e caracteriza-se por explicar os fenômenos da vida com visão reducionista. Sob influência da epistemologia cartesiana, os estudos médicos visam a compreender o funcionamento do corpo humano e as alterações anatômicas e biológicas sofridas durante a doença ${ }^{(4)}$. O corpo passa a ser o foco das práticas assistenciais e os processos terapêuticos são voltados para a identificação e o tratamento de doenças, lesões e danos. Desde a publicação do Relatório Flexner ${ }^{(5)}$, há 100 anos, nos EUA, essa visão foi assumindo papel significativo na organização dos serviços de saúde no mundo ocidental, mantendo-se hegemônica até os dias atuais.

As práticas assistenciais decorrentes desse modelo assistencial incorporaram os avanços tecnológicos em saúde, obtendo diagnósticos mais precisos, tratamento e cura de doenças complexas, alívio de muitas situações de dor e sofrimento. Por outro lado, intensificaram-se as críticas a esse modelo, incluindo o debate sobre o caráter da doença, se é essencialmente biológica ou, ao contrário, social(6), bem como sobre os limites do

1 Professora do Departamento de Enfermagem da Universidade Estadual de Maringá - UEM. Mestre em enfermagem. Doutora em enfermagem pelo Programa de PósGraduação em enfermagem da Universidade Federal de Santa Catarina - UFSC.

2 Professora do Departamento e Programa de Pós-Graduação em Enfermagem da Universidade Federal de Santa Catarina - UFSC. Doutora em ciências sociais. Pós-doutorado na University of Amsterdam. Pesquisadora do CNPq. Presidente do Conselho Regional de Enfermagem de Santa Catarina (2008-2011). 
modelo assistencial em saúde na compreensão da relação entre a dinâmica do viver e o processo saúde-doença.

No Brasil, as críticas ao modelo assistencial hegemônico geraram propostas alternativas presentes na Estratégia Saúde da Família (ESF) ${ }^{(7)}$, 1994, e na Política Nacional de Promoção da Saúde ${ }^{(8)}$, 2006. Essas políticas adotam uma concepção ampliada de saúde inspirada no que foi definido na VIII Conferência Nacional de Saúde (CNS) em 1986(9), no direito universal à saúde, como definido na Constituição Federal de $1988^{(10)}$ e nas diretrizes da política de atenção primária de saúde, formulada pela Organização Mundial da Saúde (OMS) em Alma-Ata, em 1978. A ESF se propõe a implementar um novo modelo assistencial baseado na integralidade, com foco na família e na comunidade, na vigilância em saúde e na visão de saúde como multideterminada. Já a política de Promoção da Saúde destaca a importância de promover a saúde agindo sobre os determinantes e/ou condicionantes das doenças e dos agravos à saúde, estimulando a adoção de modos de viver não violentos e o desenvolvimento de uma cultura de paz. No âmbito internacional, destaca-se a política da Safety Promotion ${ }^{(11)}$ (Promoção da Segurança) assumida pela Organização Mundial da Saúde, que considera que a segurança e as formas de prevenção de danos desenvolvidos nos espaços onde as pessoas vivem são fundamentais para ter qualidade de vida e saúde.

A multiplicidade e complexidade das condições sociais, econômicas e de estilo de vida refletem na saúde das pessoas, interferindo significativamente na demanda pelos serviços de saúde. Este cenário exige novas ações, equipamentos e intervenções, constituindo-se em um desafio para os trabalhadores/equipes e serviços de saúde ${ }^{(12)}$.

Considerando-se, de um lado, as políticas prescritas e as dificulades de implementação e, de outro, que as práticas assistenciais envolvem relações entre usuários e profissionais, é importante que as equipes de saúde considerem as concepções de saúde dos usuários. Cabe identificar se esses aspiram apenas receber cuidados para o enfrentamento de situações de doença, como prescrito na proposta da biomedicina, ou se entendem saúde de forma ampliada, como previsto nas propostas de um novo modelo assistencial.

Nesse sentido, este artigo buscou apreender, nas concepções de saúde de usuários da ESF, se essas se aproximam do modelo biomédico hegemônico ou dos princípios orientadores de um novo modelo assistencial em saúde.

\section{Metodologia}

Trata-se de um recorte de pesquisa de natureza qualitativa, realizada com usuários de cinco equipes da Estratégia Saúde da Família, de dois municípios da Região Sul do Brasil. A amostra foi composta considerando os critérios de intencionalidade, conveniência e bola de neve. Para a escolha dos grupos, foram incluídas equipes da ESF que fossem referência no atendimento em Saúde da Família e municípios com ampla disponibilidade de serviços de atenção básica e acessíveis aos sujeitos da pesquisa.

A seleção dos usuários ocorreu a partir da indicação dos Agentes Comunitários de Saúde (ACS) e de usuários entrevistados. Os critérios de inclusão foram: usuário cadastrado no Sistema de Informação da Atenção Básica (Siab) e/ou na Ficha A (instrumento de cadastro da família); receber acompanhamento contínuo da equipe da ESF pelo menos há um ano; ter participado ou participar de alguma atividade educativa ou de promoção da saúde; possuir idade superior a 18 anos; ser indicado pelos Agentes Comunitários de Saúde (ACS) ou por outro usuário no momento da entrevista (bola de neve); e aceitar participar do estudo, respeitando os critérios éticos relativos à pesquisa com seres humanos (Resolução 196/1996).

Foram selecionados 19 usuários cadastrados nas cinco equipes da ESF, que foram entrevistados entre maio e setembro de 2009. Utilizou-se a entrevista semiestruturada e roteiro com questões semiabertas, buscando identificar o conceito de saúde dos usuários. No município $A$, foram entrevistados oito usuários, identificados como $A 1, A 2, A 3 \ldots$ No município $B$, foram entrevistados 11 usuários, identificados como B1, B2, B3...

A faixa etária dos 19 participantes variou entre 25 e 77 anos de idade, com predomínio entre 40 e 55 anos, sendo 18 do sexo feminino.

A análise qualitativa foi orientada pela análise categorial temática de $\operatorname{Bardin}^{(13)}$, destacando as concepções de saúde dos usuários e as aproximações com os pressupostos do modelo biomédico ou com o novo modelo assistencial.

\section{Resultados}

A análise das entrevistas permitiu conhecer quatro principais temas representativos das concepções de saúde dos usuários da ESF: saúde como condição para a vida; saúde como qualidade de vida/bem viver; saúde como resultante de condições econômicas; saúde como ausência de doença, mas associada ao bem viver. As concepções dos usuários indicaram que, para ter saúde, não basta não ter doença, como se verifica nas diretrizes orientadoras do modelo da biomedicina; são necessárias qualidade de vida e autonomia para executar diversas atividades. Identificaram-se, também, ideias de saúde como condição para a vida e que há relação entre condição econômica e acesso aos serviços de saúde.

Na concepção de saúde como condição para a vida, ela é referida como inerente ao processo de viver. Saúde é vida e condição para o desenvolvimento das atividades cotidianas.

"Para a gente, saúde é a maior riqueza do mundo, né? Sem ela, a gente não faz nada. Se você não tiver saúde, não tem disposição para lavar uma roupa, fazer um almoço, né? Limpar a casa, cuidar de criança." B5

"Não ter saúde atrapalha tudo. A saúde é mais importante do 
que o dinheiro. Porque, se você tiver saúde, você trabalha, faz uma coisa, faz outra. Ele (dinheiro) é importante, mas a saúde é muito mais." A6

$\mathrm{Na}$ concepção de saúde como boa qualidade de vida, destacaram-se as relações entre saúde e estilos de vida saudáveis, cuidados com alimentação, exercícios físicos, bem como autonomia e condições para realizar atividades distintas.

"Saúde, para mim, é uma pessoa saudável, né? Eu acho que a pessoa tem de praticar esportes, cuidar da alimentação, não ser estressado. Ser uma pessoa com paciência. Cuidar da alimentação, do estresse do cotidiano. Sempre sorrir, quando puder não se irritar com pouco!" B11

"É a coisa mais importante, né? Porque você tem de se cuidar para não ficar doente. Cuidar da alimentação, fazer caminhadas..." A9

"Melhor coisa que tem. Ter saúde é tudo. Não ter limites para sair de casa, ir aonde quiser. Festejava muito, saía, ia a bailes, dançava, me divertia muito. Então, é ter autonomia para ir a qualquer lugar." A2

$\mathrm{Na}$ categoria saúde como ausência de doença, mas associada ao bem viver, a totalidade dos entrevistados expressam que saúde não se resume a ausência de doença, acrescentando a ideia de bem viver e de bem-estar ao entendimento e à concepção de saúde.

"Além de não ter nenhuma doença, é mais do que isso, né? Ter, assim, boas condições, assim, de viver, de lazer." B4

"Saúde é viver bem, não sentir dor (...). Estar bem." A3

"Não precisar de tratamento, medicamento, ser normal para tudo, é poder trabalhar." A4

Observa-se, sobretudo nessa categoria, que medicamentos e tratamentos, apesar de necessários, não são suficientes para ter saúde.

$\mathrm{Na}$ concepção de saúde como resultante de condições econômicas, identificaram-se discursos que relacionam saúde e boas condições econômicas para ter acesso aos serviços de saúde.

"Ser saudável. Está difícil. Se a pessoa não tiver dinheiro, não tem atendimento. É você poder trabalhar, levantar, poder ir e vir..." A8

\section{Discussão}

Na concepção de saúde referida pelos usuários, foi consensual a ideia de que saúde é mais do que ausência de doença, sinalizando congruência com as diretrizes propostas no debate atual sobre o novo modelo assistencial constante no âmbito do Sistema Único de Saúde (SUS) e em especial na Estratégia Saúde da Família e na Política Nacional de Promoção da Saúde.

A partir dos anos 1990, com a implementação do SUS, os desafios para concretizar as diretrizes que o orientam e o enfrentamento dos problemas decorrentes do modelo biomédico hegemônico em saúde geraram debates na academia e nos serviços de saúde ${ }^{(14-16)}$ e novas propostas de modelo assistencial. Nesse contexto, destaca-se a ESF como estratégia assentada no trabalho em equipe com olhar interdisciplinar e com reorientação das práticas assistenciais pautadas na família, em seu espaço de viver, na promoção e na vigilância à saúde, no diagnóstico da saúde da comunidade e nos indicadores epidemiológicos e socioeconômicos. A ESF prevê o desenvolvimento de práticas além dos muros da unidade de saúde, incluindo a visita domiciliar e internação domiciliar ${ }^{(7)}$. Essa proposta de reorientação do modelo assistencial aproxima-se das expectativas dos usuários identificadas na pesquisa.

As concepções de saúde dos usuários como qualidade de vida e bem viver aproximam-se dos pressupostos da Política Nacional de Promoção da Saúde, que foi pensada para complementar e fortalecer a Estratégia Saúde da Família. A implantação consiste em uma política integradora e dialógica com as diversas áreas do setor sanitário, compondo redes de compromisso e corresponsabilidade quanto à qualidade de vida da população, em que todos sejam partícipes na proteção e no cuidado com a vida ${ }^{(8)}$.

Essa política de saúde contempla a educação em saúde, que deve promover novas formas de abordagens, como a promoção de atividades físicas, hábitos saudáveis de alimentação e vida, controle do tabagismo e do uso abusivo de bebida alcoólica, modos de viver, condições de trabalho, habitação, ambiente, educação, lazer, cultura, acesso a bens e serviços essenciais e cuidados especiais com o processo de envelhecimento e promoção do desenvolvimento sustentável. Esses elementos compõem uma estrutura necessária para a promoção da qualidade de vida e foram sinalizados como relevantes elementos para ter saúde.

A promoção da qualidade de vida diz respeito à redução da vulnerabilidade e dos riscos à saúde, à prevenção da violência, ao estímulo à cultura de paz e à preservação do ambiente onde as pessoas vivem e trabalham, para ser mais seguro e saudável. Dialogam assim com o conceito de Safety Promotion ${ }^{(11)}$, que inclui modos de viver com maior segurança, a prevenção dos riscos/vulnerabilidades e a promoção de ambientes seguros.

As concepções de usuários sobre saúde e qualidade de vida aproximam-se, sobretudo, do conceito de saúde elaborado na VIII Conferência Nacional de Saúde em 1986, que definiu saúde como "resultante das condições de alimentação, habitação, educação, renda, meio ambiente, trabalho, transporte, emprego, tempo livre, liberdade, acesso e posse de terra e acesso aos serviços de saúde"(9:118-119).

O conceito de saúde da OMS, divulgado em 7 de abril de 1948, considera que "a saúde é o estado do mais completo bem-estar físico, mental e social, e não apenas a ausência de enfermidade"(17:539). Esse conceito gerou múltiplas críticas pela amplitude, caráter subjetivo e idealização de perfeito bem-estar, aproximando-se de uma utopia. Em decorrência dessas críticas, em 1978, na Conferência Internacional de Assistência Primária à Saúde (Alma-Ata, atual Cazaquistão), promovida pela OMS, foi aprovado um conceito ampliado 
de saúde. Enfatizaram-se as desigualdades de saúde entre países desenvolvidos e subdesenvolvidos; a responsabilidade governamental na provisão da saúde; e a importância da participação de pessoas e comunidades no planejamento e na implementação dos cuidados à saúde. Os serviços que prestam os cuidados primários de saúde representam a porta de entrada para o sistema de saúde e devem ser adaptados às condições econômicas, socioculturais e políticas da região e incluir educação em saúde, nutrição adequada, saneamento básico, planejamento familiar, imunizações, prevenção e controle de doenças endêmicas e outros agravos à saúde e provisão de medicamentos essenciais.

Bub $^{(18)}$ refere que não é possível compreender a dimensão biológica sem considerar a saúde vinculada ao bem-estar (OMS) ou ao valor social (VIII Conferência Nacional de Saúde), sendo que as duas perspectivas não são excludentes.

Os estudos da antropologia da saúde têm ressaltado que, ao pensar acerca da saúde, é importante considerar a perspectiva do usuário, uma vez que a doença tem uma dimensão cultural influenciada pela experiência subjetiva da aflição(1-3). Profissionais de saúde e usuários, mesmo quando têm a mesma origem social e cultural, podem perceber os problemas de saúde de maneiras distintas, pois as perspectivas estão baseadas em premissas diferentes. $\mathrm{O}$ universo profissional e a vivência da doença podem ter aproximações ou distanciamentos, sendo necessário refletir sobre modos de garantir a comunicação entre esses dois universos.

\section{Considerações finais}

As concepções de saúde de usuários da Estratégia Saúde da Família identificadas neste estudo mostraram um dissenso em relação às práticas assistenciais assentadas no modelo biomédico hegemônico, focadas nas doenças, na medicalização e na consulta médica, e congruência com os pressupostos discutidos na Reforma Sanitária nos anos 1980, com as diretrizes de um novo modelo assistencial em saúde, contidas na ESF, na PNPS e no conceito de saúde da VIII Conferência Nacional de Saúde.

A análise do conteúdo manifestado nas falas dos usuários sinalizou o desejo de não ter doença, possuir boa qualidade de vida, autonomia para executar diversas atividades, bem como condições econômicas para o acesso à saúde e às dimensões da vida, como trabalho, educação e lazer, que influenciam na saúde. A concepção de saúde como condição para a vida se aproxima da proposta de defesa da vida de $\operatorname{Campos}^{(19)}$, que pressupõe que as práticas assistenciais envolvem a relação entre pessoas e requerem uma responsabilização ética, política e humanitária, em direção à defesa da vida.

A identificação da perspectiva dos usuários contribuiu para mostrar o significativo valor de amplos aspectos do viver humano nas práticas em saúde. No contexto da saúde da família, as equipes de saúde necessitam estar preparadas para o enfrentamento desses aspectos, e as perspectivas dos usuários são parâmetros relevantes considerados por gestores e profissionais na organização dos serviços de saúde.

\section{Referências}

1. Helman CG. Cultura, saúde e doença. $4^{\mathrm{a}}$ ed. Porto Alegre: Artes Médicas; 2003. 2. Langdon EJ. A doença como experiência: a construção da doença e seu desafio para a prática médica [Internet]. [citado em 2010 Jun 18]. Disponível em: http://www.cfh.ufsc.br/ nessi/A\%20Doenca\%20como\%20Experiencia.htm. 3. Kleinman A. Patients and healers in the context of culture. Berdley: University of California; 1980

4. Capra F. O ponto de mutação. 22ª ed. São Paulo: Cultrix; 2001. 5. Flexner A. Medical education in the Carnegie Foundation for the advancemente of teaching. New York: Bulletin Number Four; 1910. 6. Laurell AC. A saúde-doença como processo social. In: Nunes, ED, organizador. Medicina social: aspectos históricos e teóricos. São Paulo: Global; 1983. p. 135-58.

7. Ministério da Saúde (BR). Secretaria de Assistência à Saúde. Coordenação de Saúde da Comunidade. Saúde da família: uma estratégia para reorientação do modelo assistencial. Brasília: Ministério da Saúde; 1997. 8. Ministério da Saúde (BR). Secretaria de Vigilância em Saúde. Política Nacional de Promoção da Saúde. Brasília: Ministério da Saúde; 2006. 9. Minayo MCS. A Saúde em estado de choque. Rio de Janeiro: Espaço Tempo/Fase; 1986.

10. Brasil. Constituição da República Federativa do Brasil. Brasília: Senado; 1988. 11. Svanström L. Safe communities and injury prevention: convergence in a global quest or an experiment in "empowered deliberative democracy"? J Inj Violence Prev. 2006;4(1):77-84.

12. Viana ALD, Dal Poz MR. A reforma do sistema de saúde no Brasil e o Programa de Saúde da Família. Physis. 2005;15(Supl.):225-64. 13. Bardin L. Análise de conteúdo. Lisboa: Edições 70; 2002. 14. Fertonani HP. O desafio de construir um novo modelo assistencial em saúde: reflexões de trabalhadores do PSF de uma unidade de saúde de Maringá-PR [dissertação]. Florianópolis: Universidade Federal de Santa Catarina; 2003.

15. Pires D. Reestruturação produtiva e trabalho em saúde no Brasil. $2^{\mathrm{a}}$ ed. São Paulo: Confederação Nacional dos Trabalhadores em Seguridade Social/ Annablume; 2008.

16. Cecílio LCO. Modelos tecno-assistenciais em saúde: da pirâmide ao círculo, uma possibilidade a ser explorada. Cad Saúde Pública. 1997;13(3):469-78.

17. Segre M, Ferraz FC. O conceito de saúde. Rev Saúde Pública. 1997;31(5): $538-42$.

18. Bub MBC. Concepções de saúde, ética e prática de enfermagem [tese]. Florianópolis: Universidade Federal de Santa Catarina; 2001.

19. Campos GWS. A saúde pública e a defesa da vida. $2^{a}$ ed. São Paulo: Hucitec; 1994. 